\title{
FIXED POINT INDEX APPROACH FOR SOLUTIONS OF VARIATIONAL INEQUALITIES
}

\author{
YISHENG LAI, YUANGUO ZHU, AND YINBING DENG
}

Received 8 October 2004 and in revised form 18 May 2005

By using fixed point index approach for multivalued mappings, the existence of nonzero solutions for a class of generalized variational inequalities is studied in reflexive Banach space. One of the mappings concerned here is coercive or monotone and the other is set-contractive or upper semicontinuous.

\section{Introduction}

Since the fundamental theory of variational inequality was founded in the 1960s, the variational inequality theory with applications has made powerful progress and has become an important part of nonlinear analysis. It has been applied intensively to mechanics, differential equation, cybernetics, quantitative economics, optimization theory, nonlinear programming, and so forth (see [2]).

In virtue of minimax theorem of Ky Fan and KKM technique, variational inequalities, generalized variational inequalities, and generalized quasivariational inequalities were studied intensively in the last 20 years with topological method, variational method, semiordering method, and fixed point method [2]. However, the existence of nonzero solutions for variational inequalities, as another important topic of variational inequality theory, has been rarely discussed.

It is of theoretical and practical significance to study the existence of nonzero solutions for variational inequalities. In this paper, we will discuss the existence of nonzero solutions for a class of generalized variational inequalities for multivalued mappings by fixed point index approach in reflexive Banach space.

Let $Y, Z$ be two topological spaces. A multivalued mapping $F: Y \rightarrow 2^{Z}$ is called upper semicontinuous at $y_{0} \in Y$ if for each neighbourhood $V \subset Z$ of $F\left(y_{0}\right)$, there exists a neighbourhood $U$ of $y_{0}$ such that the set $F(U) \subset V$. Suppose that $E_{1}, E_{2}$ are two real Banach spaces, $D \subseteq E_{1}$. A multivalued mapping $A: D \rightarrow 2^{E_{2}}$ is said to be $k$-set-contractive on $D$ if there exists a constant $k$ such that $\alpha(A(S)) \leq k \alpha(S)$ whenever $\alpha(S) \neq 0, S \subseteq D$, where $\alpha$ is the Kuratowski measure of noncompactness. A mapping $A$ is called condensing on $D$ if $\alpha(A(S))<\alpha(S)$ whenever $\alpha(S) \neq 0, S \subseteq D$. It is easily seen that a mapping $A$ is condensing when $k<1$. Let $X$ be a Banach space, $X^{*}$ its dual, and $(\cdot, \cdot)$ the pair between $X^{*}$ 
and $X$. Suppose that $K$ is a closed convex subset of $X$ and $U$ is an open subset of $X$ with $U_{K}=U \cap K \neq \varnothing$. The closure and boundary of $U_{K}$ relative to $K$ are denoted by $\overline{U_{K}}$ and $\partial\left(U_{K}\right)$, respectively. Assume that $T: \overline{U_{K}} \rightarrow 2^{K}$ is an upper semicontinuous mapping with nonempty compact convex values and $T$ is also condensing. If $x \notin T(x)$ for $x \in \partial\left(U_{K}\right)$, then the fixed point index, $i_{K}(T, U)$, is well defined (see [3]).

Proposition 1.1 [3]. Let $K$ be a nonempty closed convex subset of a real Banach space $X$ and let $U$ be an open subset of $X$. Suppose that $T: \overline{U_{K}} \rightarrow 2^{K}$ is an upper semicontinuous mapping with nonempty compact convex values and $x \notin T(x)$ for $x \in \partial\left(U_{K}\right)$, then the index, $i_{K}(T, U)$, has the following properties:

(i) if $i_{K}(T, U) \neq 0$, then $T$ has a fixed point;

(ii) for mapping $\hat{X}_{0}$ with constant value $\left\{x_{0}\right\}$, if $x_{0} \in U_{K}$, then $i_{K}\left(\hat{X}_{0}, U\right)=1$;

(iii) let $U_{1}, U_{2}$ be two open subsets of $X$ with $U_{1} \cap U_{2}=\varnothing$. If $x \notin T(x)$ when $x \in$ $\partial\left(\left(U_{1}\right)_{K}\right) \cup \partial\left(\left(U_{2}\right)_{K}\right)$, then $i_{K}\left(T, U_{1} \cup U_{2}\right)=i_{K}\left(T, U_{1}\right)+i_{K}\left(T, U_{2}\right) ;$

(iv) let $H:[0,1] \times \overline{U_{K}} \rightarrow 2^{K}$ be an upper semicontinuous mapping with nonempty compact convex values and $\alpha(H([0,1] \times Q))<\alpha(Q)$ whenever $\alpha(Q) \neq 0, Q \subseteq \overline{U_{K}}$. If $x \notin H(t, x)$ for every $t \in[0,1], x \in \partial\left(U_{K}\right)$, then $i_{K}(H(1, \cdot), U)=i_{K}(H(0, \cdot), U)$.

In this paper, for mappings $A: X \rightarrow X^{*}$ and $g: K \rightarrow 2^{X^{*}}$, we will deal with the following problem by fixed point index approach: find $u \in K, u \neq 0$, and $w \in g(u)$ such that

$$
(A u, v-u) \geq(w, v-u), \quad \forall v \in K
$$

\section{Nonzero solutions when the mapping $A$ is coercive}

Suppose that $K$ is a subspace of $X$ and $A: X \rightarrow X^{*}$ is a coercive and linear continuous mapping, that is, there exist constants $M, \gamma>0$ such that

$$
(A v, v) \geq \gamma\|v\|^{2}, \quad\|A v\|_{X^{*}} \leq M\|v\|, \quad \forall v \in X .
$$

It is well known that for any given $w \in X^{*}$, the variational inequality

$$
(A u, v-u) \geq(w, v-u), \quad \forall v \in K
$$

has an only solution $u$ in $K$ (see [1]). Define a mapping as follows:

$$
K_{A}: X^{*} \longrightarrow K, \quad K_{A}(w)=u, \quad \forall w \in X^{*},
$$

then $K_{A}$ is a coercive and linear continuous mapping and (see [1])

$$
\left\|K_{A}\left(w_{1}\right)-K_{A}\left(w_{2}\right)\right\| \leq \frac{1}{\gamma}\left\|w_{1}-w_{2}\right\|_{X^{*}}
$$


Theorem 2.1. Let $K$ be a subspace of a reflexive real Banach space X. Suppose that $A$ : $X \rightarrow X^{*}$ is a coercive and linear continuous mapping which satisfies inequalities (2.1) and $g: K \rightarrow 2^{X^{*}}$ is $\beta$-set-contractive and upper semicontinuous mapping with nonempty compact convex values, where $\beta / \gamma<1$. Assume

(a) $\liminf _{\left\|u_{n}\right\| \rightarrow 0} \sup _{w_{n} \in g\left(u_{n}\right)}\left(w_{n}, u_{n}\right) /\left\|u_{n}\right\|^{2}<\gamma\left(u_{n} \in K\right)$;

(b) there exist $x_{0} \in K$ and a constant $q>0$ such that $\inf _{w \in g(u)}\left(w, x_{0}\right) /\|u\|>M\left\|x_{0}\right\|$ when $\|u\|>q, u \in K$.

Then (1.1) has a nonzero solution.

Proof. Define a mapping as follows:

$$
K_{A} g: K \longrightarrow 2^{K}, \quad\left(K_{A} g\right)(u)=K_{A}(g(u)), \quad \forall u \in K
$$

It is easily seen that $K_{A} g$ is $(\beta / \gamma)$-set-contractive and upper semicontinuous mapping with nonempty compact convex values by (2.4). Let $K^{r}=\{x \in K,\|x\|<r\}$. Assuming that there does not exist $r \neq 0$ and $u \in \partial\left(\overline{K^{r}}\right)$ such that $u \in K_{A}(g(u)$ ) (or else $u$ is a nonzero solution of (1.1)). We will verify that $i_{K}\left(K_{A} g, K^{r}\right)=1$ for small enough $r$ and $i_{K}\left(K_{A} g, K^{R}\right)=0$ for large enough $R$.

Firstly, define a mapping by $H:[0,1] \times \overline{K^{r}} \rightarrow 2^{K}, H(t, u)=t K_{A}(g(u))$. Obviously, $H(t, u)$ is an upper semicontinuous mapping with nonempty compact convex values. We claim that $\alpha(H([0,1] \times Q))<\alpha(Q)$ whenever $\alpha(Q) \neq 0, Q \subseteq \overline{K^{r}}$. In fact, let $e \in K_{A} g(Q)$, then $0 \in\left\{K_{A} g(Q)-e\right\}$. Hence, we have

$$
\begin{aligned}
H([0,1] \times Q) & =\bigcup_{t \in[0,1]}\left\{t\left[K_{A} g(Q)-e\right]+t e\right\} \\
& \subseteq \bigcup_{t \in[0,1]}\left\{t\left[K_{A} g(Q)-e\right]\right\}+\bigcup_{t \in[0,1]}\{t e\} \\
& \subseteq\left\{K_{A} g(Q)-e\right\}+\bigcup_{t \in[0,1]}\{t e\} .
\end{aligned}
$$

Thus

$$
\alpha(H([0,1] \times Q)) \leq \alpha\left(\left\{K_{A} g(Q)\right\}\right)+\alpha\left(\bigcup_{t \in(0,1)}\{t e\}\right)=\alpha\left(\left\{K_{A} g(Q)\right\}\right)<\alpha(Q) .
$$

We claim that there exists small enough $r$ such that $u \notin H(t, u)$ for all $t \in[0,1], u \in \partial\left(K^{r}\right)$. Otherwise, there exist two sequences $\left\{t_{n}\right\},\left\{u_{n}\right\}, t_{n} \in(0,1], u_{n} \in \partial\left(K^{r}\right),\left\|u_{n}\right\| \rightarrow 0$, such that $u_{n} \in H\left(t_{n}, u_{n}\right)=t_{n} K_{A} g\left(u_{n}\right)$ or $u_{n} / t_{n} \in K_{A} g\left(u_{n}\right)$, hence there exists $w_{n} \in g\left(u_{n}\right)$ such that $u_{n} / t_{n}=K_{A}\left(w_{n}\right)$, that is, we have

$$
\left(A\left(\frac{u_{n}}{t_{n}}\right), v-\frac{u_{n}}{t_{n}}\right) \geq\left(w_{n}, v-\frac{u_{n}}{t_{n}}\right), \quad \forall v \in K
$$

Letting $v=0$, we can obtain from (2.1) and (2.8) that

$$
\gamma \leq \frac{\left(A u_{n}, u_{n}\right)}{\left\|u_{n}\right\|^{2}} \leq t_{n} \frac{\left(w_{n}, u_{n}\right)}{\left\|u_{n}\right\|^{2}} \leq \frac{\left(w_{n}, u_{n}\right)}{\left\|u_{n}\right\|^{2}} .
$$


Thus liminf $\left\|u_{n}\right\| \rightarrow 0 \sup _{w_{n} \in g\left(u_{n}\right)}\left(w_{n}, u_{n}\right) /\left\|u_{n}\right\|^{2} \geq \gamma$, which contradicts condition (a). Therefore,

$$
i_{K}\left(K_{A} g, K^{r}\right)=i_{K}\left(H(1, \cdot), K^{r}\right)=i_{K}\left(H(\cdot, 0), K^{r}\right)=i_{K}\left(\hat{0}, K^{r}\right)=1
$$

by Proposition 1.1(ii) and (iv).

Secondly, we will verify that $i_{K}\left(K_{A} g, K^{R}\right)=0$ for large enough $R$. In fact, we can get from (2.1) and condition (b) that

$$
\left(w, x_{0}\right)>\left(A u, x_{0}\right), \quad \forall w \in g(u), \text { as }\|u\|>q .
$$

On the other hand, because $g$ is $\beta$-set-contractive and upper semicontinuous mapping with nonempty compact convex values, there exists a constant $L>0$ such that $\|w\|_{X^{*}} \leq L$ for all $w \in g(u)$ whenever $\|u\| \leq q, u \in K$. Take $N$ for large enough and $f \in X^{*}$ so that

$$
M q\left\|x_{0}\right\|+L\left\|x_{0}\right\|<-N\left(f, x_{0}\right) .
$$

Define a mapping by $H:[0,1] \times \overline{K^{R}} \rightarrow 2^{K}, H(t, u)=K_{A}(g(u)-t N f)$. Then $H(t, u)$ is an upper semicontinuous mapping with nonempty compact convex values. We claim that $\alpha(H([0,1] \times Q))<\alpha(Q)$ whenever $\alpha(Q) \neq 0, Q \subseteq \overline{K^{r}}$. In fact,

$$
\begin{gathered}
H([0,1] \times Q)=K_{A}\left(\bigcup_{t \in[0,1]}\{g(Q)-t N f\}\right) \subseteq K_{A}\left(\{g(Q)\}+\bigcup_{t \in[0,1]}\{-t N f\}\right), \\
\alpha\left(\{g(Q)\}+\bigcup_{t \in[0,1]}\{-t N f\}\right) \leq \alpha(g(Q))+\alpha\left(\bigcup_{t \in[0,1]}\{-N t f\}\right)=\alpha(g(Q)) \leq \beta \alpha(Q) .
\end{gathered}
$$

Thus $\alpha(H([0,1] \times Q)) \leq(\beta / \gamma) \alpha(Q)<\alpha(Q)$ by $(2.4)$ and $\beta / \gamma<1$. We claim that there exists large enough $R$ such that $u \notin H(t, u)$ for all $t \in[0,1], u \in \partial\left(\overline{K^{R}}\right)$. Otherwise, there exist two sequences $\left\{t_{n}\right\},\left\{u_{n}\right\}, t_{n} \in[0,1], u_{n} \in \partial\left(\overline{K^{R}}\right),\left\|u_{n}\right\| \rightarrow+\infty$, such that $u_{n} \in H\left(t_{n}\right.$, $\left.u_{n}\right)=K_{A}\left(g\left(u_{n}\right)-t_{n} N f\right)$, hence there exists $w_{n} \in g\left(u_{n}\right)$ such that $u_{n}=K_{A}\left(w_{n}-t_{n} N f\right)$, that is, we have

$$
\left(A u_{n}, v-u_{n}\right) \geq\left(w_{n}-t_{n} N f, v-u_{n}\right), \quad \forall v \in K .
$$

Taking $v=u_{n}+x_{0}$ in (2.14), we obtain from (2.1) that

$$
M\left\|x_{0}\right\| \geq \frac{\left(A u_{n}, x_{0}\right)}{\left\|u_{n}\right\|} \geq \frac{\left(w_{n}, x_{0}\right)}{\left\|u_{n}\right\|} \geq \inf _{w_{n} \in g\left(u_{n}\right)} \frac{\left(w_{n}, x_{0}\right)}{\left\|u_{n}\right\|},
$$

which contradicts condition (b). Therefore,

$$
i_{K}\left(K_{A} g, K^{R}\right)=i_{K}\left(H(\cdot, 0), K^{R}\right)=i_{K}\left(H(1, \cdot), K^{R}\right)
$$

by Proposition $1.1\left(\right.$ iv). If $i_{K}\left(H(1, \cdot), K^{R}\right) \neq 0$, then the mapping $H(1, \cdot): K \rightarrow 2^{K}$ has a fixed point $u$ in $K^{R}$ by Proposition 1.1(i), that is, $u \in H(1, u)=K_{A}(g(u)-N f)$. Thus there exists $w \in g(u)$ such that $u=K_{A}(w-N f)$, that is,

$$
(A u, v-u) \geq(w-N f, v-u), \quad \forall v \in K .
$$


Taking $v=u+x_{0}$ in (2.17), we get that

$$
\left(A u, x_{0}\right)-\left(w, x_{0}\right) \geq-N\left(f, x_{0}\right) .
$$

That contradicts (2.11) if $\|u\|>q$, hence $\|u\| \leq q$, then we can get from (2.1) and (2.18) that

$$
-N\left(f, x_{0}\right) \leq\left|\left(A u, x_{0}\right)\right|+\left|\left(w, x_{0}\right)\right| \leq M q\left\|x_{0}\right\|+L\left\|x_{0}\right\|
$$

but it contradicts $(2.12)$. Therefore, $i_{K}\left(H(1, \cdot), K^{R}\right)=0$.

It follows from (2.10), (2.16), and Proposition 1.1(iii) that $i_{K}\left(K_{A} g, K^{R} \backslash \overline{K^{r}}\right)=-1$. Therefore, there exists a fixed point $u \in K^{R} \backslash \overline{K^{r}}$ which is a nonzero solution of (1.1).

Theorem 2.2. Let $K$ be a subspace of a reflexive real Banach space $X$. Suppose that $A$ : $X \rightarrow X^{*}$ is a coercive and linear continuous mapping which satisfies inequalities (2.1) and $g: K \rightarrow 2^{X^{*}}$ is $\beta$-set-contractive and upper semicontinuous mapping with nonempty compact convex values, where $\beta / \gamma<1$. Assume

(a) $\liminf _{\left\|u_{n}\right\| \rightarrow+\infty} \sup _{w_{n} \in g\left(u_{n}\right)}\left(w_{n}, u_{n}\right) /\left\|u_{n}\right\|^{2}<\gamma\left(u_{n} \in K\right)$;

(b) there exist $x_{0} \in K$ and an open neighbourhood $V(0)$ of zero point such that for any given $u \in K \cap V(0) \backslash\{0\}, \inf _{w \in g(u)}\left(w, x_{0}\right) /\|u\|>M\left\|x_{0}\right\|$.

Then (1.1) has a nonzero solution.

The proof of Theorem 2.2 is similar to that of Theorem 2.1. We omit it here.

\section{Nonzero solutions when the mapping $A$ is monotone}

Let $A: X \rightarrow X^{*}$ be a monotone linear mapping with $(A u, u) /\|u\| \rightarrow+\infty$ (as $\|u\| \rightarrow+\infty$, $u \in K)$. It is well known that for any given $w \in X^{*}$, the variational inequality (2.2) has solutions in $K$ (see [2]), thus we may define two mappings as follows:

$K_{A}: X^{*} \longrightarrow 2^{K}, \quad K_{A}(w)=\{u \in K: u$ is a solution of the variational inequality (2.2) $\}$,

$$
K_{A} g: K \longrightarrow 2^{K}, \quad\left(K_{A} g\right)(u)=K_{A}(g(u)), \quad \forall u \in K
$$

Proposition 3.1. Let $X=R^{n}$ and let $K \subset X$ be a nonempty closed convex set. Suppose that $A: X \rightarrow X^{*}$ is a monotone hemicontinuous mapping. If for every $w \in X^{*}$, the variational inequality (2.2) has solutions in $K$, then the mapping $K_{A}$ in (3.1) is a monotone and upper semicontinuous mapping with nonempty compact convex values.

Proof. Let $u_{1} \in K_{A}\left(w_{1}\right), u_{2} \in K_{A}\left(w_{2}\right)$. Then

$$
\left(A u_{i}, v-u_{i}\right) \geq\left(w_{i}, v-u_{i}\right), \quad \forall v \in K, i=1,2
$$

It is easily obtained from above inequalities that $\left(A u_{1}-A u_{2}, u_{1}-u_{2}\right) \leq\left(w_{1}-w_{2}, u_{1}-u_{2}\right)$. Thus $K_{A}$ is monotone due to the monotony of $A$. Furthermore, $K_{A}$ is locally bounded by [3]. We claim that $K_{A}$ is upper semicontinuous. Otherwise, there exists a point $w \in X^{*}$ 


\section{Solutions of variational inequalities}

and an open set $V_{0}$ containing $K_{A}(w)$ such that for sequence $\left\{w_{n}\right\}$ converging to $w$, there exist $u_{n} \in K_{A}\left(w_{n}\right)$ such that $u_{n} \notin V_{0}$. Since $\left\{u_{n}\right\}$ is bounded by the locally boundedness of $K_{A}$, there exists a subsequence $\left\{u_{n_{k}}\right\}$ such that $u_{n_{k}} \rightarrow u_{0}$. Obviously, $u_{0} \in K, u_{0} \notin V_{0}$. We know that monotone hemicontinuous mapping $A$ is continuous by [3]. Letting $k \rightarrow+\infty$ in the inequality

$$
\left(A u_{n_{k}}, v-u_{n_{k}}\right) \geq\left(w_{n_{k}}, v-u_{n_{k}}\right), \quad \forall v \in K,
$$

yields that

$$
\left(A u_{0}, v-u_{0}\right) \geq\left(w, v-u_{0}\right), \quad \forall v \in K
$$

which implies that $u_{0} \in K_{A}(w) \subset V_{0}$. That is a contradiction. In addition, the compact convexity of $K_{A}(w)$ is obvious.

Proposition 3.2. Let $K$ be a subspace of a reflexive real Banach space $X$. Suppose that $A: X \rightarrow X^{*}$ is a monotone linear mapping with $(A u, u) /\|u\| \rightarrow+\infty($ as $\|u\| \rightarrow+\infty, u \in K)$ and $g: K \rightarrow 2^{X^{*}}$ is a mapping with nonempty convex values, then $K_{A} g: K \rightarrow 2^{K}$ (3.2) is also a mapping with nonempty convex values.

Proof. Let $q \in K$ and $u_{1}, u_{2} \in K_{A} g(q)$. Then there exist $w_{1}, w_{2} \in g(q)$ such that $u_{i} \in K_{A}\left(w_{i}\right)$, $i=1,2$. That is, we have

$$
\begin{array}{ll}
\left(A u_{1}, v-u_{1}\right) \geq\left(w_{1}, v-u_{1}\right), & \forall v \in K, \\
\left(A u_{2}, v-u_{2}\right) \geq\left(w_{2}, v-u_{2}\right), & \forall v \in K .
\end{array}
$$

Substituting $v+u_{1}-\left(\lambda_{1} u_{1}+\lambda_{2} u_{2}\right)$ (resp., $\left.v+u_{2}-\left(\lambda_{1} u_{1}+\lambda_{2} u_{2}\right)\right)$ for $v$ in (3.6) (resp., in (3.7)), where $\lambda_{1}, \lambda_{2} \geq 0, \lambda_{1}+\lambda_{2}=1$, we get that

$$
\left(\lambda_{1} A u_{1}+\lambda_{2} A u_{2}, v-\sum_{i=1}^{2} \lambda_{i} u_{i}\right) \geq\left(\lambda_{1} w_{1}+\lambda_{2} w_{2}, v-\sum_{i=1}^{2} \lambda_{i} u_{i}\right), \quad \forall v \in K
$$

In addition, $\sum_{i=1}^{2} \lambda_{i} w_{i} \in g(q)$. Therefore, $\sum_{i=1}^{2} \lambda_{i} u_{i} \in K_{A} g(q)$ which implies that $K_{A} g$ is a mapping with nonempty convex values.

We first consider the nonzero solutions of (1.1) in $R^{n}$.

Theorem 3.3. Let $K$ be a subspace of $X=R^{n}$. Suppose that $A: X \rightarrow X^{*}$ is a monotone linear mapping with $(A u, u) /\|u\| \rightarrow+\infty($ as $\|u\| \rightarrow+\infty, u \in K)$ and $g: K \rightarrow 2^{X^{*}}$ is an upper semicontinuous mapping with nonempty compact convex values. The following conditions either $(a),(b)$ or $\left(a^{\prime}\right),\left(b^{\prime}\right)$ are assumed to be satisfied:

(a) there exist $y_{0} \in K$ and an open neighbourhood $V(0)$ of zero point such that for any given $u \in K \cap V(0) \backslash\{0\}, \inf _{w \in g(u)}\left(A u-w, y_{0}\right)>0$;

(b) there exist $x_{0} \in K$ and a constant $q>0$ such that $\sup _{w \in g(u)}\left(A u-w, x_{0}\right)<0$ when $\|u\|>q, u \in K$

$\left(\mathrm{a}^{\prime}\right)$ there exist $y_{0} \in K$ and an open neighbourhood $V(0)$ of zero point such that for any given $u \in K \cap V(0) \backslash\{0\}$, $\sup _{w \in g(u)}\left(A u-w, y_{0}\right)<0$; 
$\left(\mathrm{b}^{\prime}\right)$ there exist $x_{0} \in K$ and a constant $q>0$ such that $\inf _{w \in g(u)}\left(A u-w, x_{0}\right)>0$ when $\|u\|>q, u \in K$.

Then (1.1) has a nonzero solution.

Proof. It is well known that monotone linear mapping must be semicontinuous (see [2]), hence $K_{A}: X^{*} \rightarrow 2^{K}$ (3.1) is an upper semicontinuous mapping with nonempty compact convex values by Proposition 3.1. It is easy to see from [2] that $K_{A} g: K \rightarrow 2^{K}$, $\left(K_{A} g\right)(u)=K_{A}(g(u)), u \in K$, is an upper semicontinuous mapping with nonempty compact values, therefore $K_{A} g$ is an upper semicontinuous mapping with nonempty compact convex values by Proposition 3.2.

Let $K^{r}=\{x \in K,\|x\|<r\}$. Similar to the proof of Theorem 2.1, we may get that $i_{K}\left(K_{A} g, K^{R} \backslash \overline{K^{r}}\right)=-1$. Therefore, there exists a fixed point $u \in K^{R} \backslash \overline{K^{r}}$ which is a nonzero solution of (1.1).

Now, we discuss the nonzero solution of (1.1) in reflexive real Banach space.

Theorem 3.4. Let $K$ be a subspace of a reflexive real Banach space X. Suppose that $A$ : $X \rightarrow X^{*}$ is a monotone linear mapping with $(A u, u) /\|u\| \rightarrow+\infty($ as $\|u\| \rightarrow+\infty, u \in K)$ and $g: K \rightarrow 2^{X^{*}}$ is an upper semicontinuous from the weak topology on $X$ to the strong topology on $X^{*}$, with nonempty compact convex values. The following conditions either $(a),(b),(c)$ or $\left(a^{\prime}\right),\left(b^{\prime}\right),(c)$ are assumed to be satisfied:

(a) there exist $y_{0} \in K$ and an open neighbourhood $V(0)$ of zero point such that for any given $u \in K \cap V(0) \backslash\{0\}, \inf _{w \in g(u)}\left(A u-w, y_{0}\right)>0$;

(b) there exist $x_{0} \in K$ and a constant $q>0$ such that $\sup _{w \in g(u)}\left(A u-w, x_{0}\right)<0$ when $\|u\|>q, u \in K$

(c) there exists $z_{0} \in K$ such that liminf ${ }_{u_{\alpha}}{ }_{\rightarrow}{ }_{0} \sup _{w \in g\left(u_{\alpha}\right)}\left(A u_{\alpha}-w, z_{0}\right)<0$, where $u_{\alpha} \in K$;

$\left(\mathrm{a}^{\prime}\right)$ there exist $y_{0} \in K$ and an open neighbourhood $V(0)$ of zero point such that for any given $u \in K \cap V(0) \backslash\{0\}, \sup _{w \in g(u)}\left(A u-w, y_{0}\right)<0$;

$\left(\mathrm{b}^{\prime}\right)$ there exist $x_{0} \in K$ and a constant $q>0$ such that $\inf _{w \in g(u)}\left(A u-w, x_{0}\right)>0$ when $\|u\|>q, u \in K$.

Then (1.1) has a nonzero solution.

Proof. Let $F \subset X$ be a finite-dimensional subspace containing $x_{0}, y_{0}$, and $z_{0}$. We will show that all conditions in Theorem 3.3 are satisfied on space $F$. Denote $K_{F}=K \cap F$. Let $j_{F}: F \rightarrow X$ be an injective mapping and $j_{F}^{*}: X^{*} \rightarrow F^{*}$ its dual mapping. Denote $A_{F}=$ $j_{F}^{*}\left(A \mid K_{F}\right): K_{F} \rightarrow F^{*}, g_{F}=j_{F}^{*}\left(g \mid K_{F}\right): K_{F} \rightarrow F^{*}$. We know that $A_{F}=j_{F}^{*} A j_{F}, g_{F}=j_{F}^{*} g j_{F}$. Then $A_{F}, g_{F}$ are linear and upper semicontinuous with nonempty compact convex values, respectively. For $x_{1}, x_{2}, u \in K_{F}$, we have

$$
\begin{aligned}
&\left(A_{F}\left(x_{1}\right)-A_{F}\left(x_{2}\right), x_{1}-x_{2}\right)=\left(j_{F}^{*} A\left(x_{1}\right)-j_{F}^{*} A\left(x_{2}\right), x_{1}-x_{2}\right) \\
&=\left(A\left(x_{1}\right)-A\left(x_{2}\right), j_{F}^{*}\left(x_{1}-x_{2}\right)\right) \\
&=\left(A\left(x_{1}\right)-A\left(x_{2}\right), x_{1}-x_{2}\right) \geq 0 \\
& \frac{\left(A_{F}(u), u\right)}{\|u\|}=\frac{\left(j_{F}^{*} A(u), u\right)}{\|u\|}=\frac{(A u, u)}{\|u\|} .
\end{aligned}
$$


These mean that $A_{F}$ is monotone with $\left(A_{F}(u), u\right) /\|u\| \rightarrow+\infty$ (as $\left.\|u\| \rightarrow+\infty, u \in K_{F}\right)$. On the other hand,

$$
\begin{aligned}
\inf _{w \in g_{F}(u)}\left(A_{F}(u)-w, y_{0}\right) & =\inf _{w \in j^{*} g(u)}\left(j_{F}^{*} A(u)-w, y_{0}\right) \\
& =\inf _{w^{\prime} \in g(u)}\left(j_{F}^{*} A(u)-j_{F}^{*}\left(w^{\prime}\right), y_{0}\right) \\
& =\inf _{w^{\prime} \in g(u)}\left(A(u)-w^{\prime}, y_{0}\right), \\
\sup _{w \in g_{F}(u)}\left(A_{F}(u)-w, x_{0}\right) & =\sup _{w^{\prime} \in g(u)}\left(A(u)-w^{\prime}, x_{0}\right) .
\end{aligned}
$$

Therefore, there exists $u_{F}, u_{F} \neq 0$, and $w_{F}^{\prime} \in g_{F}\left(u_{F}\right)$ from conditions (a) and (b) or ( $\left.\mathrm{a}^{\prime}\right)$ and $\left(b^{\prime}\right)$ and Theorem 3.3 such that

$$
\left(A_{F}\left(u_{F}\right), v-u_{F}\right) \geq\left(w_{F}^{\prime}, v-u_{F}\right), \quad \forall v \in K_{F} .
$$

Since $w_{F}^{\prime} \in g_{F}\left(u_{F}\right)=j^{*}\left(g\left(u_{F}\right)\right)$, there exists $w_{F} \in g\left(u_{F}\right)$ such that $w_{F}^{\prime}=j_{F}^{*}\left(w_{F}\right)$. Hence,

$$
\left(A\left(u_{F}\right), v-u_{F}\right) \geq\left(w_{F}, v-u_{F}\right), \quad \forall v \in K_{F},
$$

by (3.11). Suppose that conditions (a) and (b) are satisfied, taking $v=u_{F}+x_{0}$ (or else $\left.v=u_{F}-x_{0}\right)$, we get that $\left(A\left(u_{F}\right)-w_{F}, x_{0}\right) \geq 0$. Thus $\sup _{w \in g\left(u_{F}\right)}\left(A\left(u_{F}\right)-w, x_{0}\right) \geq 0$, this conduces to a contradiction by condition (b) if $\left\|u_{F}\right\| \rightarrow+\infty$. Hence, there exists a constant $M>0$ such that $\left\|u_{F}\right\| \leq M$ for all finite-dimensional subspace $F$ containing $x_{0}, y_{0}$, and $z_{0}$. Since $X$ is reflexive and $K$ is weakly closed, there exists $u^{\prime} \in K$ such that for every finite-dimensional subspace $F$ containing $x_{0}, y_{0}$, and $z_{0}, u^{\prime}$ is in the weak closure of the set $V_{F}=\bigcup_{F \subset F_{1}}\left\{u_{F_{1}}\right\}$, where $F_{1}$ is a finite-dimensional subspace in $X$. In fact, because $V_{F}$ is bounded, we know that $\overline{\left(V_{F}\right)^{w}}$ (the weak closure of the set $V_{F}$ ) is weakly compact. On the other hand, let $F^{1}, F^{2}, \ldots, F^{m}$ be finite-dimensional subspace containing $x_{0}$, $y_{0}$, and $z_{0}$. Set $F^{(m)}:=\operatorname{span}\left\{F^{1}, F^{2}, \ldots, F^{m}\right\}$. Then $F^{(m)}$, which contains $x_{0}, y_{0}$, and $z_{0}$, is a finite-dimensional subspace. Hence, $\bigcap_{i=1}^{m} V_{F^{i}}=\bigcap_{i=1}^{m}\left(\bigcup_{F^{i} \subset F_{1}}\left\{u_{F_{1}}\right\}\right)=\bigcup_{F^{(m)} \subset F_{1}}\left\{u_{F_{1}}\right\} \neq \varnothing$ and then $\bigcap_{F} \overline{\left(V_{F}\right)^{w}} \neq \varnothing$. Now let $v \in K$ and let $F^{\prime}$ be a finite-dimensional subspace which contains $x_{0}, y_{0}, z_{0}$ and $v$. Since $u^{\prime}$ belongs to the weak closure of the set $V_{F^{\prime}}=\bigcup_{F^{\prime} \subset F_{1}}\left\{u_{F_{1}}\right\}$, we may find a sequence $\left\{u_{F_{\alpha}}\right\}$ in $V_{F^{\prime}}$ such that $u_{F_{\alpha}} \stackrel{w}{\rightarrow} u^{\prime}$. There exists a sequence $\left\{w_{F_{\alpha}}\right\}$, $w_{F_{\alpha}} \in g\left(u_{F_{\alpha}}\right)$, from (3.12) such that $\left(A\left(u_{F_{\alpha}}\right), v-u_{F_{\alpha}}\right) \geq\left(w_{F_{\alpha}}, v-u_{F_{\alpha}}\right)$. Because $g: K \rightarrow 2^{X^{*}}$ is an upper semicontinuous from the weak topology on $X$ to the strong topology on $X^{*}$, there exist $w^{\prime} \in g\left(u^{\prime}\right)$ and a subsequence $\left\{w_{F_{\beta}}\right\} \subset\left\{w_{F_{\alpha}}\right\}$ by $[2,5]$ such that the sequence $\left\{w_{F_{\beta}}\right\} \stackrel{s}{\rightarrow} w^{\prime}$ (strongly converges to $w^{\prime}$ ). However, $u_{F_{\beta}}$ and $w_{F_{\beta}}$ satisfy the following inequality:

$$
\left(A\left(u_{F_{\beta}}\right), v-u_{F_{\beta}}\right) \geq\left(w_{F_{\beta}}, v-u_{F_{\beta}}\right) .
$$

The monotony of $A$ implies that

$$
\left(A(v), v-u_{F_{\beta}}\right) \geq\left(w_{F_{\beta}}, v-u_{F_{\beta}}\right) .
$$


Letting $u_{F_{\beta}} \stackrel{w}{\rightarrow} u^{\prime}$ and $\left\{w_{F_{\beta}}\right\} \stackrel{s}{\rightarrow} w^{\prime}$ yields that

$$
\left(A v, v-u^{\prime}\right) \geq\left(w^{\prime}, v-u^{\prime}\right), \quad \forall v \in K
$$

Thus

$$
\left(A\left(u^{\prime}\right), v-u^{\prime}\right) \geq\left(w^{\prime}, v-u^{\prime}\right), \quad \forall v \in K
$$

by the Minity theorem $[2,4]$. We claim that $u^{\prime} \neq 0$. Otherwise $u_{F_{\beta}} \stackrel{w}{\rightarrow} 0$. Taking $v=z_{0}+u_{F_{\beta}}$ in $(3.13)$ yields that $\left(A\left(u_{F_{\beta}}\right), z_{0}\right) \geq\left(w_{F_{\beta}}, z_{0}\right)$. Thus

$$
\sup _{w_{F_{\beta}} \in g\left(u_{F_{\beta}}\right)}\left(A\left(u_{F_{\beta}}\right)-w_{F_{\beta}}, z_{0}\right) \geq 0,
$$

which contradicts condition (c). Therefore, $u^{\prime}$ is a nonzero solution of (1.1).

\section{Acknowledgments}

This project was supported by The Natural Science Foundation of Zhejiang Province Grant no. Y104149. The authors would like to thank the anonymous referees for the helpful suggestions.

\section{References}

[1] F. E. Browder, Nonlinear monotone operators and convex sets in Banach spaces, Bull. Amer. Math. Soc. 71 (1965), 780-785.

[2] S. S. Chang, Variational Inequality and Complementarity Problem Theory with Applications, Shanghai Scientific Technology and Literature Press, Shanghai, 1991.

[3] P. M. Fitzpatrick and W. V. Petryshyn, Fixed point theorems and the fixed point index for multivalued mappings in cones, J. London Math. Soc. (2) 12 (1975/76), no. 1, 75-85.

[4] D. Kinderlehrer and G. Stampacchia, An Introduction to Variational Inequalities and Their Applications, Pure and Applied Mathematics, vol. 88, Academic Press, New York, 1980.

[5] C. H. Su and V. M. Sehgal, Some fixed point theorems for condensing multifunctions in locally convex spaces, Proc. Amer. Math. Soc. 50 (1975), 150-154.

Yisheng Lai: College of Statistics and Computer Science, Zhejiang Gongshang University, Hangzhou 310035, China

E-mail address: laiyisheng67@163.com

Yuanguo Zhu: Department of Applied Mathematics, Nanjing University of Science and Technology, Nanjing 210094, China

E-mail address: ygzhu@mail.njust.edu.cn

Yinbing Deng: Department of Mathematics, Central China Normal University, Wuhan 430079, China

E-mail address: ybdeng@public.wh.hb.cn 


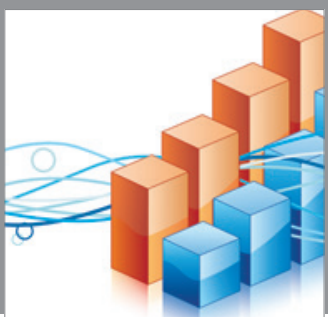

Advances in

Operations Research

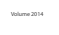



\section{The Scientific} World Journal
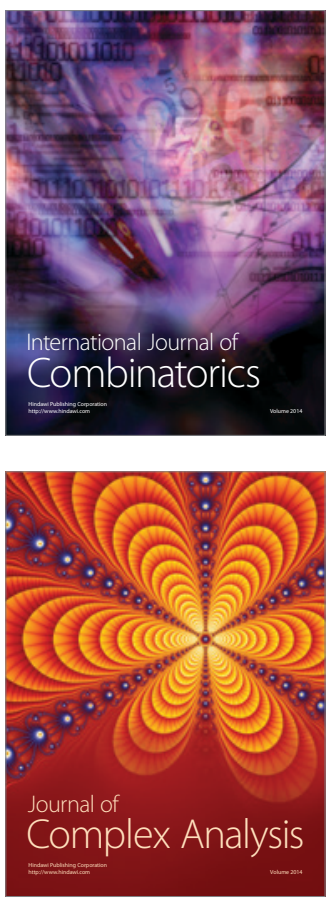

International Journal of

Mathematics and

Mathematical

Sciences
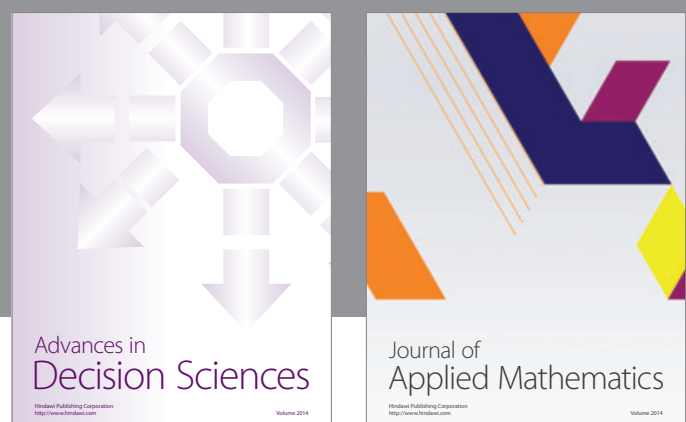

Journal of

Applied Mathematics
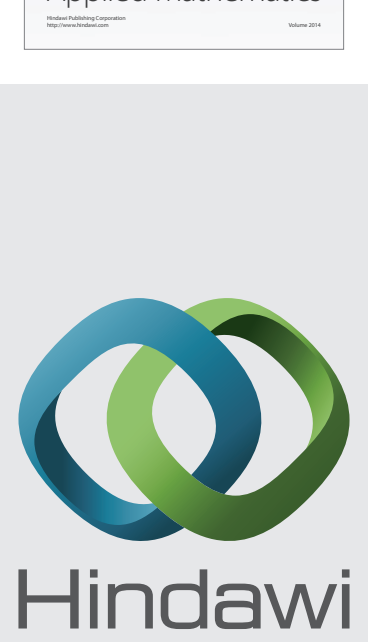

Submit your manuscripts at http://www.hindawi.com


Mathematical Problems in Engineering
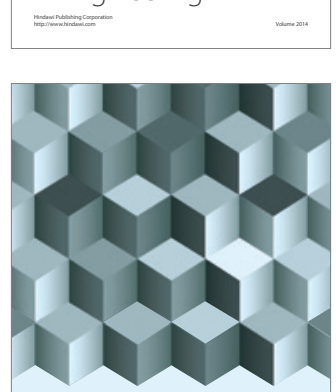

Journal of

Function Spaces
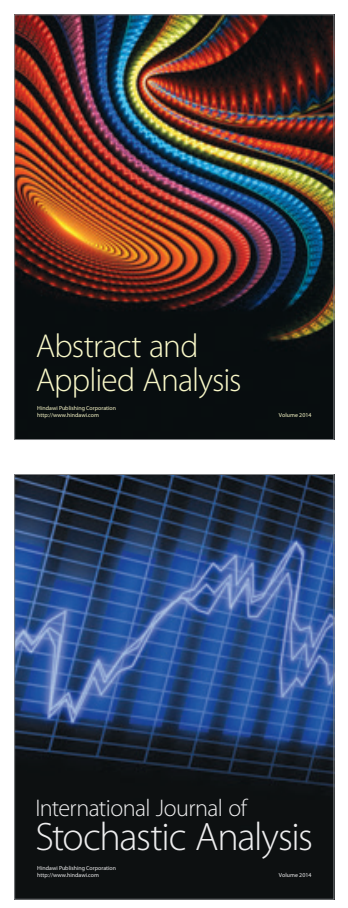

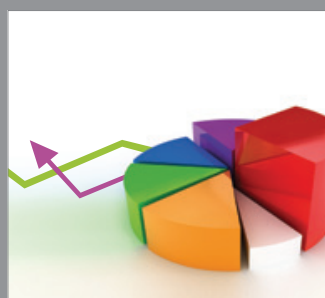

ournal of

Probability and Statistics

Promensencen
